\title{
NONDETECTION OF THE NEPTUNE-MASS PLANET REPORTED AROUND GJ 176*
}

\author{
R. Paul Butler ${ }^{1}$, Andrew W. Howard ${ }^{2,3,6}$, Steven S. Vogt ${ }^{4}$, and Jason T. Wright 5 \\ ${ }^{1}$ Department of Terrestrial Magnetism, Carnegie Institution of Washington, 5241 Broad Branch Road NW, Washington, DC 20015-1305, USA \\ ${ }^{2}$ Department of Astronomy, University of California, Berkeley, CA 94720-3411, USA \\ ${ }^{3}$ Space Sciences Laboratory, University of California, Berkeley, CA 94720-7450, USA \\ ${ }^{4}$ UCO/Lick Observatory, University of California at Santa Cruz, Santa Cruz, CA 95064, USA \\ ${ }^{5}$ Department of Astronomy, Cornell University, Ithaca, NY 14853, USA \\ Received 2008 May 26; accepted 2008 October 15; published 2009 February 9
}

\begin{abstract}
Endl et al. reported a Neptune-mass planet in a 10.24 day orbit around GJ 176. This planet has raised interest because of its low mass $\left(M \sin i=24 M_{\text {Earth }}\right)$, correspondingly small velocity amplitude $\left(K=11.7 \mathrm{~m} \mathrm{~s}{ }^{-1}\right)$, and because GJ 176 is an M star. We report 41 precise Doppler measurements of GJ 176 obtained with the Keck-HIRES spectrometer over a 10 year time span. These measurements show no evidence of the 10.24 day companion, at a threshold of $4 \mathrm{~m} \mathrm{~s}^{-1}$, a factor of 3 less than the amplitude reported by Endl et al. The Keck velocities are consistent with instrumental noise and stellar jitter. The existence of the planet is thus called into question.
\end{abstract}

Key words: planetary systems - stars: individual (GJ 176) - techniques: radial velocities

\section{INTRODUCTION}

Endl et al. (2008) reported the detection of an $M \sin i=$ $24 M_{\text {Earth }}$ planet in a 10.24 day orbit around GJ 176 (HD 285968, HIP 21932, LHS 196), a nearby M2.5 dwarf. They observed the star as part of their M dwarf planet search (Endl et al. 2003, 2006) using the High-Resolution Spectrograph (Tull 1998) on the Hobby-Eberly Telescope (HET). Their 28 velocities span 3.9 years (2003 October to 2007 September) with an rms scatter of $9.8 \mathrm{~m} \mathrm{~s}^{-1}$ and an average measurement uncertainty of $4.7 \mathrm{~m} \mathrm{~s}^{-1}$.

The reported planet is noteworthy for several reasons. Of the $\sim 290$ announced exoplanets, it is one of only a handful orbiting an $\mathrm{M}$ dwarf. The Doppler semiamplitude $\left(K=11.7 \mathrm{~m} \mathrm{~s}^{-1}\right)$ is one of the smallest reported velocity signatures to date. The minimum mass and orbital period distinguish it from the nowcommonplace hot-Jupiter class of planets. These characteristics also make GJ 176 an important data point in statistical tests of low-mass planet formation theories (e.g., Ida \& Lin 2005, 2008; Kennedy et al. 2006; Goldreich et al. 2004).

GJ 176 has also been under observation at $2 \mathrm{~m} \mathrm{~s}^{-1}$ precision for the past 10 years at the Keck I Telescope by the CaliforniaCarnegie exoplanet group, as part of the M dwarf survey (Butler et al. 2004). These observations offer the opportunity for confirmation of the planet's existence and orbital characteristics. This paper seeks to address two questions. (1) Is there evidence in the Keck observations of GJ 176 for a 10.24 day planet, as reported by Endl et al.? (2) Are the quality and timing of the Keck observations sufficient to have detected the planet? Section 2 describes the radial velocity observations of GJ 176 taken with the Keck Telescope, as well as a variety of analyses in search of the 10.24 day planet. Our nondetection of the GJ 176 companion is discussed in Section 3.

\footnotetext{
* Based on observations obtained at the W. M. Keck Observatory, which is operated jointly by the University of California and the California Institute of Technology. Keck time has been granted by both NASA and the University of California.

6 Townes Fellow.
}

\section{KECK DOPPLER VELOCITIES AND PERIODICITIES}

\subsection{Observations}

GJ 176 was observed using the High-Resolution Echelle Spectrometer (HIRES; Vogt et al. 1994) on the $10 \mathrm{~m}$ Keck I Telescope. We used the well known Doppler velocity technique described by Butler et al. (1996). Forty-one velocities were collected between 1998 January and 2008 March with an rms scatter of $7.4 \mathrm{~m} \mathrm{~s}^{-1}$ and a median measurement uncertainty of $1.9 \mathrm{~m} \mathrm{~s}^{-1}$ (Table 1 and Figure 1). Compared to the HET data there are nearly $50 \%$ more Keck velocities, with smaller uncertainties, spanning more than twice the time period.

The true velocity variability of GJ 176-an admixture of orbital companion signatures and stellar jitter-can be estimated for a given set of observations as the quadrature difference of the rms scatter $\left(7.4 \mathrm{~m} \mathrm{~s}^{-1}\right.$ for Keck, $9.8 \mathrm{~m} \mathrm{~s}^{-1}$ for HET) and the typical measurement uncertainty $\left(1.9 \mathrm{~m} \mathrm{~s}^{-1}\right.$ for Keck, ${ }^{7}$ $4.7 \mathrm{~m} \mathrm{~s}^{-1}$ for HET). Our estimate of this quantity from the Keck data $\left(7.1 \mathrm{~m} \mathrm{~s}^{-1}\right)$ is smaller than, but comparable to, the Texas group's estimate from the HET data set $\left(8.6 \mathrm{~m} \mathrm{~s}^{-1}\right)$. Thus the $\mathrm{rms}$ velocity variation caused by jitter and any planets is similar from both instruments.

In the analysis that follows, we binned the Keck velocities into $2 \mathrm{hr}$ time bins to average over some short-timescale nonKeplerian sources of variability. The binning only affects the last four velocities in Table 1, reducing the effective number of Keck velocities from 41 to 39.

Figures 2-4 show the radial velocities of a representative sample of $12 \mathrm{M}$ dwarfs with $B-V$ between 1.46 and 1.59 , and $V$ magnitude between 9.56 and 10.75, similar to GJ 176 .

\footnotetext{
7 Each velocity measurement is calculated from the Doppler shift of the observed stellar spectrum with respect to a fixed iodine transmission spectrum. The relevant portion of the spectrum (5000-6200 Å) is divided into $\sim 700$ chunks, with each chunk producing an estimate for the Doppler shift and an associated error. The mean of this distribution and the associated error in determining the mean are the reported Keck radial velocity and measurement uncertainty for a given spectrum. While it is not straightforward to disentangle the sources of measurement uncertainty, they include instrumental/modeling (systematic) sources-imperfect optics and photodetectors, inadequate point-spread function (PSF) characterization, undersampled stellar and iodine spectra-and physical sources—photon statistics, stellar oscillations. For further details, see Butler et al. (1996).
} 


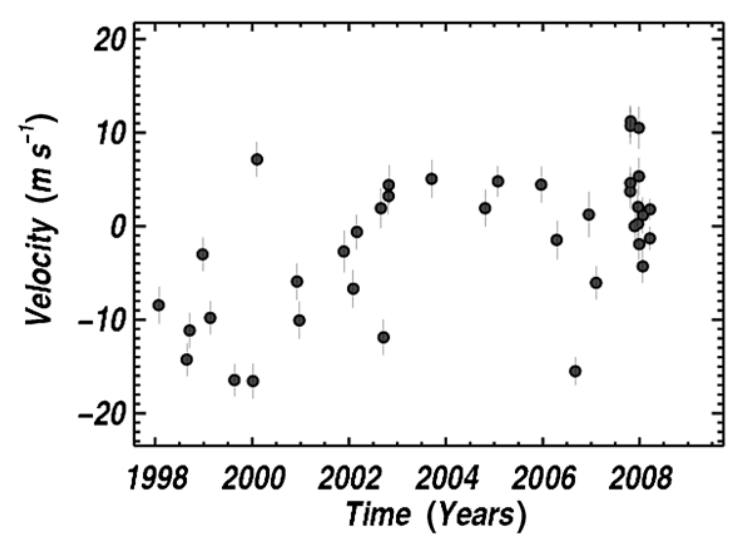

Figure 1. Radial velocity time series for GJ 176 from Keck-HIRES observations with measurement uncertainties shown. The velocities have an rms scatter of $7.4 \mathrm{~m} \mathrm{~s}^{-1}$

Table 1

Radial Velocities for GJ 176 from the Keck Telescope

\begin{tabular}{|c|c|c|}
\hline $\mathrm{JD}-2,440,000$ & $\begin{array}{c}\text { Radial Velocity } \\
\left(\mathrm{m} \mathrm{s}^{-1}\right)\end{array}$ & $\begin{array}{c}\text { Uncertainty } \\
\left(\mathrm{m} \mathrm{s}^{-1}\right)\end{array}$ \\
\hline 10839.760 & -8.45 & 2.0 \\
\hline 11051.083 & -14.26 & 1.8 \\
\hline 11073.109 & -11.15 & 1.9 \\
\hline 11170.901 & -3.02 & 1.8 \\
\hline 11228.780 & -9.80 & 1.8 \\
\hline 11411.129 & -16.45 & 1.7 \\
\hline 11550.867 & -16.55 & 1.9 \\
\hline 11581.854 & 7.14 & 1.9 \\
\hline 11882.996 & -5.92 & 2.0 \\
\hline 11900.995 & -10.07 & 2.0 \\
\hline 12235.842 & -2.70 & 2.2 \\
\hline 12307.810 & -6.69 & 2.0 \\
\hline 12334.771 & -0.62 & 1.9 \\
\hline 12516.133 & 1.92 & 2.1 \\
\hline 12536.081 & -11.89 & 1.9 \\
\hline 12573.943 & 3.21 & 1.9 \\
\hline 12575.039 & 4.40 & 2.1 \\
\hline 12898.124 & 5.05 & 2.0 \\
\hline 13302.090 & 1.92 & 2.0 \\
\hline 13398.894 & 4.79 & 1.6 \\
\hline 13724.957 & 4.43 & 1.9 \\
\hline 13841.743 & -1.47 & 2.1 \\
\hline 13982.057 & -15.50 & 1.5 \\
\hline 14084.046 & 1.24 & 2.4 \\
\hline 14138.895 & -6.06 & 1.8 \\
\hline 14396.883 & 3.72 & 1.9 \\
\hline 14397.931 & 4.60 & 1.7 \\
\hline 14398.973 & 10.72 & 1.9 \\
\hline 14399.987 & 11.20 & 1.7 \\
\hline 14430.015 & 0.00 & 2.1 \\
\hline 14454.919 & 0.25 & 2.0 \\
\hline 14455.917 & 2.05 & 2.0 \\
\hline 14460.831 & 10.51 & 2.2 \\
\hline 14461.892 & 5.32 & 2.0 \\
\hline 14464.891 & -1.92 & 1.7 \\
\hline 14490.838 & -4.30 & 1.7 \\
\hline 14491.853 & 1.16 & 1.9 \\
\hline 14544.784 & -2.33 & 1.8 \\
\hline 14544.791 & -0.41 & 1.7 \\
\hline 14545.761 & 3.41 & 1.7 \\
\hline 14545.770 & 0.37 & 1.6 \\
\hline
\end{tabular}

The velocity rms of these stars range from 3 to $7 \mathrm{~m} \mathrm{~s}^{-1}$, which includes contributions from photon limited uncertainty, stellar

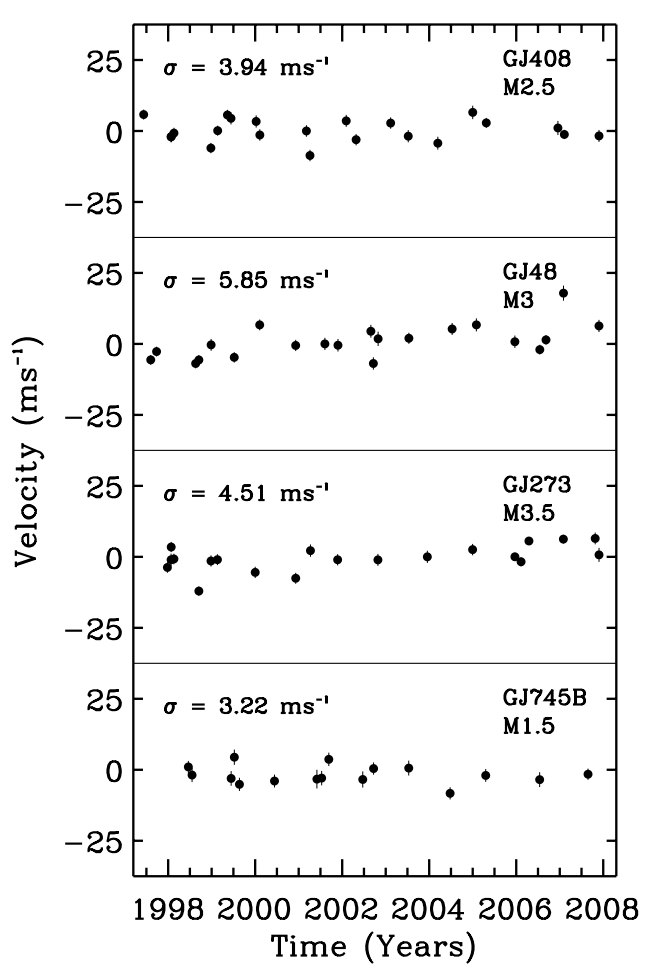

Figure 2. Radial velocity time series for four stable M dwarfs in our Keck Doppler survey that are similar to GJ 176.

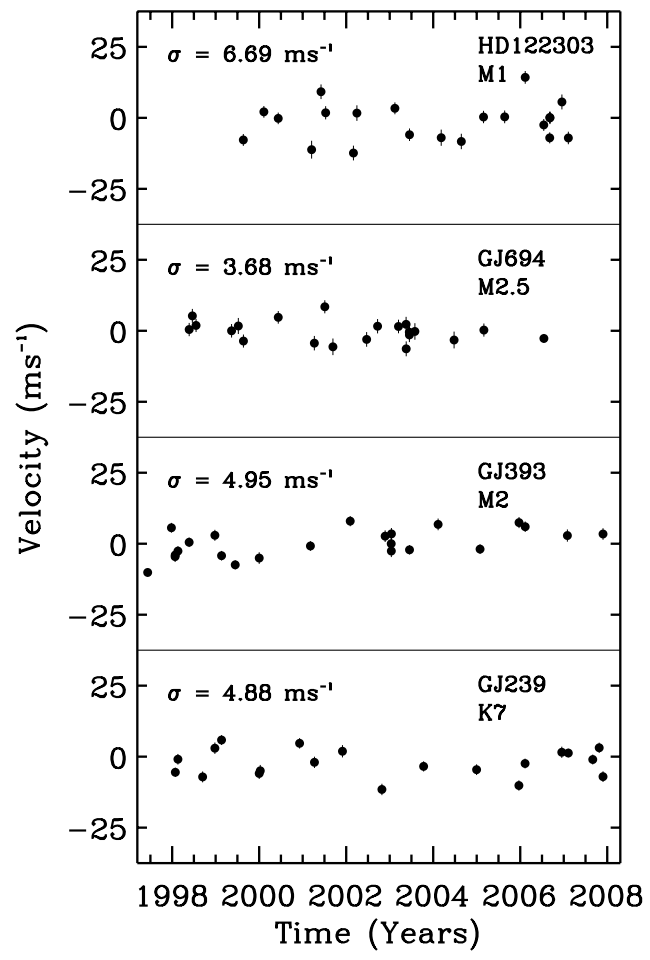

Figure 3. Radial velocity time series for a further four stable M dwarfs in our Keck Doppler survey that are similar to GJ 176.

jitter, undiscovered planets, and remaining systematic errors in our velocity reduction package. Figure 5 shows the $\mathrm{Ca}$ II $\mathrm{H}$ emission for stars in Figures 2-4, as well as for GJ 176. The emission of GJ 176 is obvious to the eye, but smaller than the emission of some of the stable stars in Figure 4. These figures establish the precision of the Keck velocity measurements in $\mathrm{M}$ dwarfs as well as the jitter typical of these stars. In the analysis 


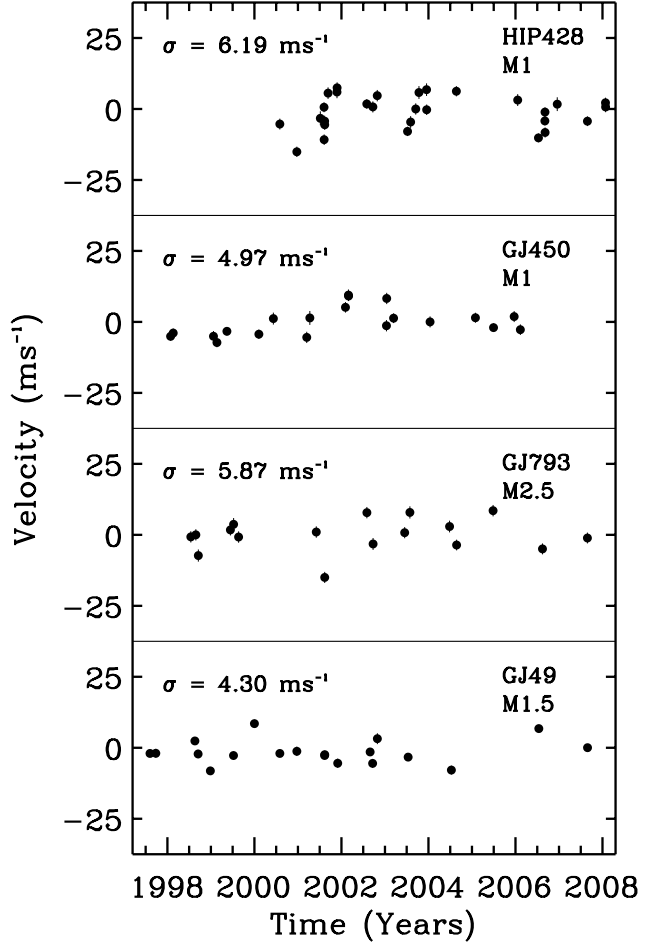

Figure 4. Radial velocity time series for a further four stable $M$ dwarfs in our Keck Doppler survey that are similar to GJ 176.

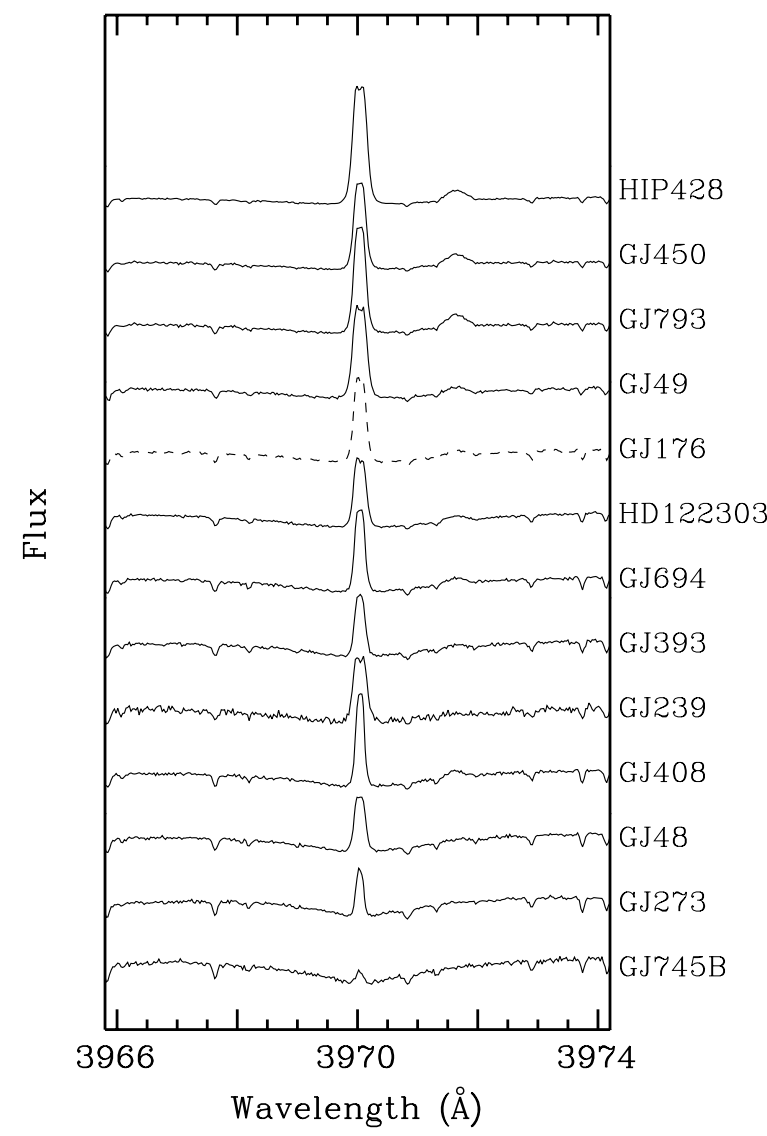

Figure 5. Spectra near the Ca II $\mathrm{H}$ line for GJ 176 (dashed line) and the stars in Figures 2, 3, and 4 (solid lines). The emission reversals reflect stellar magnetic activity that correlates with photospheric velocity jitter.

that follows, we adopt a jitter of $5 \mathrm{~m} \mathrm{~s}^{-1}$, which gives a total error of $5.3 \mathrm{~m} \mathrm{~s}^{-1}$ when added in quadrature with the typical measurement uncertainty of $1.9 \mathrm{~m} \mathrm{~s}^{-1}$.
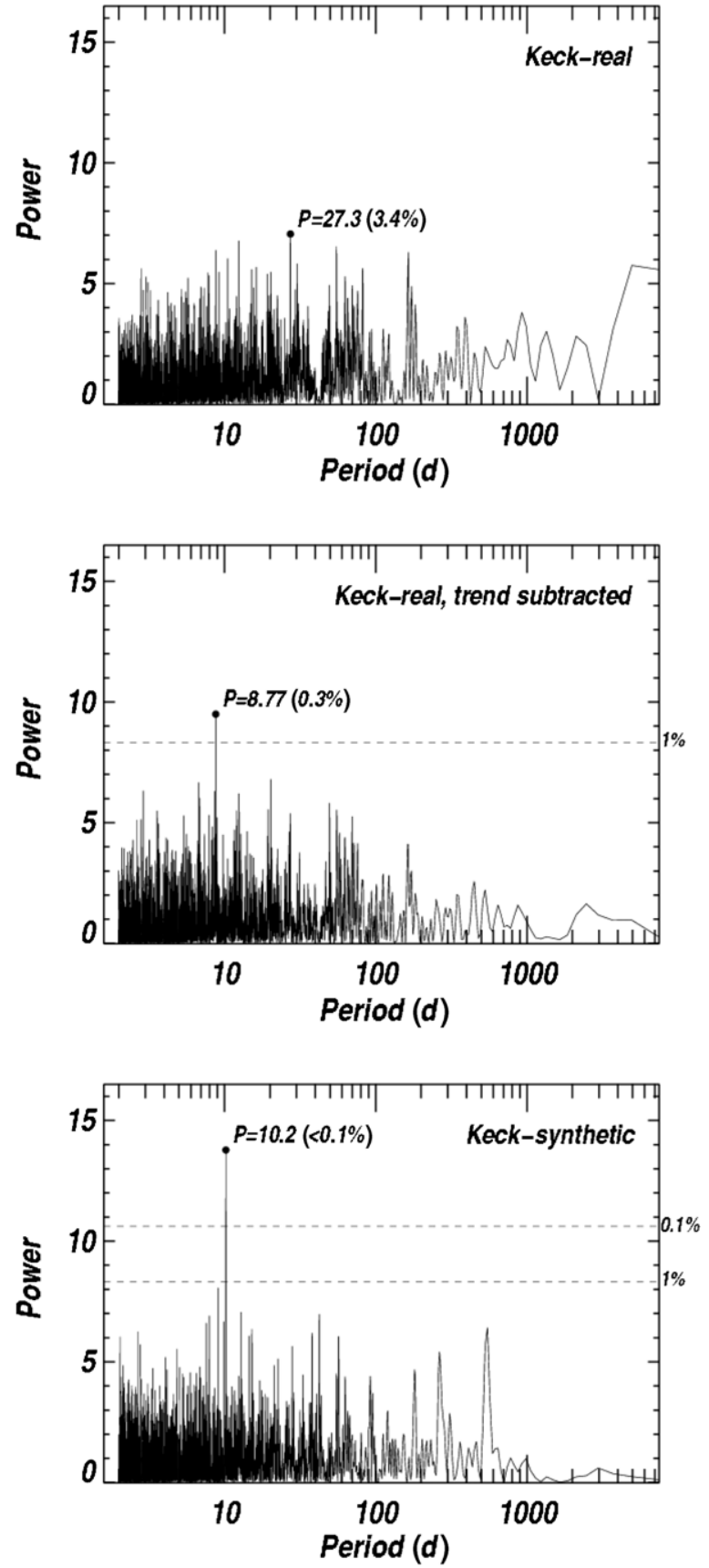

Figure 6. Periodograms of GJ 176 from the actual Keck velocities (top and middle) and synthetic Keck velocities (bottom). A linear trend of $1.2 \mathrm{~m} \mathrm{~s}^{-1} \mathrm{yr}^{-1}$ was subtracted from the real Keck velocities before the periodogram in the middle panel was calculated, while the top panel represents the velocities before detrending. The period of the highest peak in each plot is labeled and the analytic $1 \%$ and $0.1 \%$ FAP thresholds are shown where specific peaks exceed those levels. The Keck data are capable of detecting the 10.24 day planet reported by Endl et al., but do not.

\subsection{Incompatibility with the 10 day Planet}

Figure 6 (top panel) shows the Lomb-Scargle periodogram of the Keck velocities. Importantly, the Keck velocities do not have appreciable power (exceeding the 1\% false alarm level) near 10.2 days. To account for the possibility of a linear velocity trend that might hide the 10.2 day signal the middle panel of Figure 6 shows the periodogram of the same Keck velocities after subtracting a best-fit $1.2 \mathrm{~m} \mathrm{~s}^{-1} \mathrm{yr}^{-1}$ linear trend. We address the peak at 8.77 days and the significance of the linear trend in Section 2.3 . 

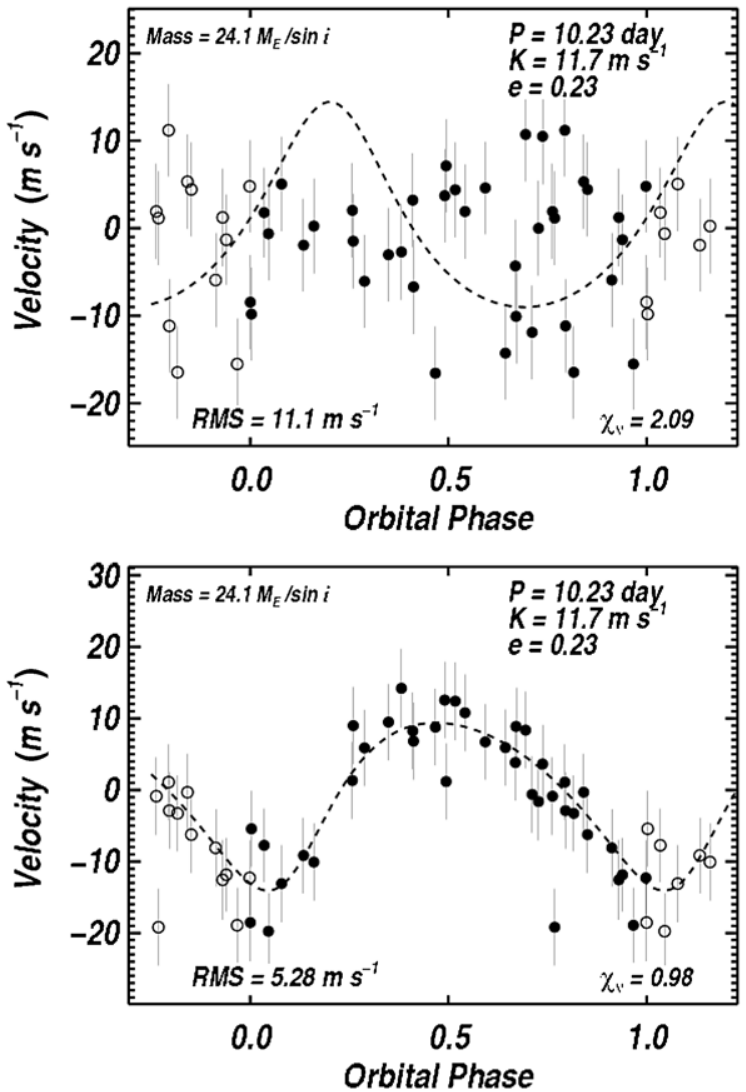

Figure 7. Best-fit Keplerian solution to the actual (top) and synthetic (bottom) phased Keck-HIRES velocities. The overplotted orbital solutions were found holding $P, K$, and $e$ fixed at their Endl et al. values $\left(T_{p}\right.$ and $\omega$, and $\gamma$ were unconstrained and converged on different values in the two cases).

The failure to detect the 10.24 day periodogram signal is significant since Keck-HIRES could easily have detected the planetary signature, as shown by the periodogram of a synthetic set of Keck velocities (Figure 6, bottom panel). These synthetic velocities have the same observation times as the actual Keck observations, and the velocities are the sum of the reported planet velocity signature (using the Endl et al. orbital parameters) and $5.3 \mathrm{~m} \mathrm{~s}^{-1}$ Gaussian noise (simulating jitter and measurement errors). The peak at 10.2 days is clearly detected in the synthetic Keck velocities. The failure to see this peak in the actual velocities is in stark conflict with the planetary hypothesis for the 10.2 day peak in the HET periodogram. Nevertheless, the sine-wave treatment of periodogram analysis is hardly the definitive test to rule a planet in or out. A full Keplerian analysis and a study of the observational phase function (to check for well timed orbits hidden in the Keck data) are required.

To test the hypothesis that the Keck-HIRES velocities are consistent with the Endl et al. orbital solution, we plot the two in the top panel of Figure 7 . The velocity curve uses the noncircular ${ }^{8}$ Endl et al. values for $P, K$, and $e$. The values of $T_{p}$ and $\omega$ are poorly determined by the modest number of HET observations and may not match the Keck velocities, which have a longer time baseline. These parameters were therefore fit to the Keck velocities using a Levenberg-Marquardt minimization. The reduced $\chi$ value $\left(\chi_{v} \equiv \sqrt{\chi_{v}^{2}}\right)$ of 2.09 for the

\footnotetext{
8 Endl et al. published two orbital solutions, one circular and the other mildly eccentric ( $e=0.232 \pm 0.127)$. These orbits have nearly identical best-fit values of $P, K, M \sin i$, and $a$. Unless otherwise noted, we use the noncircular orbital parameters, although our conclusions are largely independent of this choice.
}

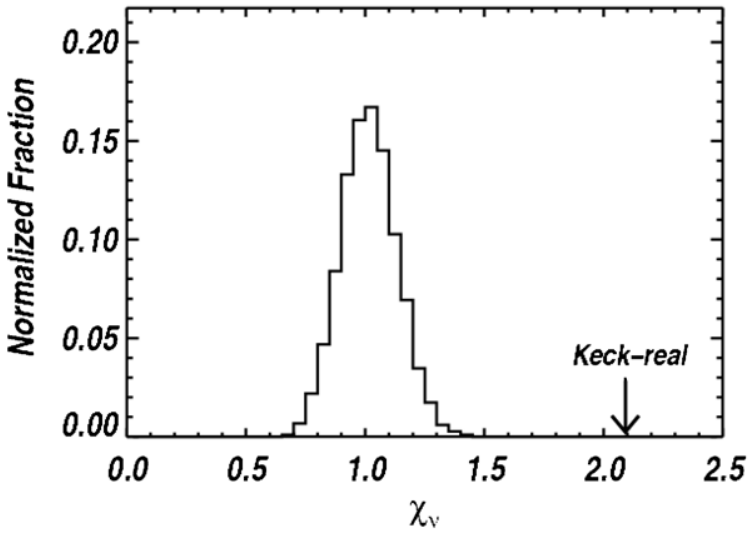

Figure 8. Histogram of $\chi_{v}$ for synthetic Keck velocities, consisting of an artificial 10.2366 day planet and noise, fit with a Keplerian model. As expected, $\chi_{v}$ is distributed near unity. In contrast, $\chi_{v}$ for the actual Keck data (indicated by the arrow) is clearly much larger than unity, arguing against the planet hypothesis. Each set of synthetic velocities was calculated as the sum of the 10.24 day planet velocities at the actual Keck observation times and $5.3 \mathrm{~m} \mathrm{~s}^{-1}$ Gaussian noise. Each value of $\chi_{v}$ in the histogram was calculated from a best-fit Keplerian solution to a set of synthetic velocities with the fit constrained to the Endl et al. values of $P, e$, and $K$, but unconstrained in $T_{p}$ and $\omega$.

Keplerian fit to the real Keck velocities confirms what is clear by visual inspection-the Keck-HIRES velocities are strongly inconsistent with the Endl et al. orbital solution.

In contrast, the bottom panel of Figure 7 shows a good orbital fit with $\chi_{v} \approx 1$ for mock Keck-HIRES observations. The synthetic velocities plotted in this panel were constructed as above using $5.3 \mathrm{~m} \mathrm{~s}^{-1}$ Gaussian noise for errors. We conclude that the temporal sampling and smaller uncertainties of the Keck-HIRES velocities are more than sufficient to detect and characterize the planet described by Endl et al.

We also confirmed that the good fit of the synthetic Keck-HIRES velocities shown in the bottom panel of Figure 7 is representative of the fit obtained from members of an ensemble of similar synthetic velocity sets. Figure 8 shows the distribution of $\chi_{v}$ from Keplerian-fitting of $10^{4}$ synthetic velocity sets (constructed as above). Each value of $\chi_{v}$ was calculated from a best-fit Keplerian solution to a different synthetic velocity set with the fit constrained to the Endl et al. values of $P, e$, and $K$, but unconstrained in $T_{p}$ and $\omega$. The distribution of $\chi_{v}$ is tightly clustered around unity, completely separated from $\chi_{v}$ $=2.09$ obtained from the actual Keck velocities. This implies that the poor fit to the actual Keck-HIRES velocities (Figure 7, top panel) is not a statistical fluke, but reflects a significant inconsistency between Keck velocities and the HET orbit.

It remains possible that the true period of the planet differs slightly from $P=10.2366$ days as reported by Endl et al. We investigated this possibility by fitting the actual Keck-HIRES velocities with Keplerians constrained to specific periods. Planet periods ranging from 9 to 11 days were systematically attempted in steps of $5 \times 10^{-5}$ days. Since this tests a specific hypothesisthe presence of a planet with the orbital parameters reported by Endl et al. - the fits were also constrained to the Endl et al. values of $e$ and $K$, while $T_{p}$ and $\omega$ were left unconstrained. ${ }^{9}$ Thus, this test is not constructed to rule out all possible planets, just a small set of planets with orbital parameters matching the hypothesis being tested.

\footnotetext{
9 A Keplerian fit to velocities spanning many orbital cycles is much more sensitive to the value of $P$ than to the values of $e$ and $K$ (because errors in $P$ are accumulated). It is therefore unnecessary to fit the real Keck data with a range
} of values of $e$ and $K$. 


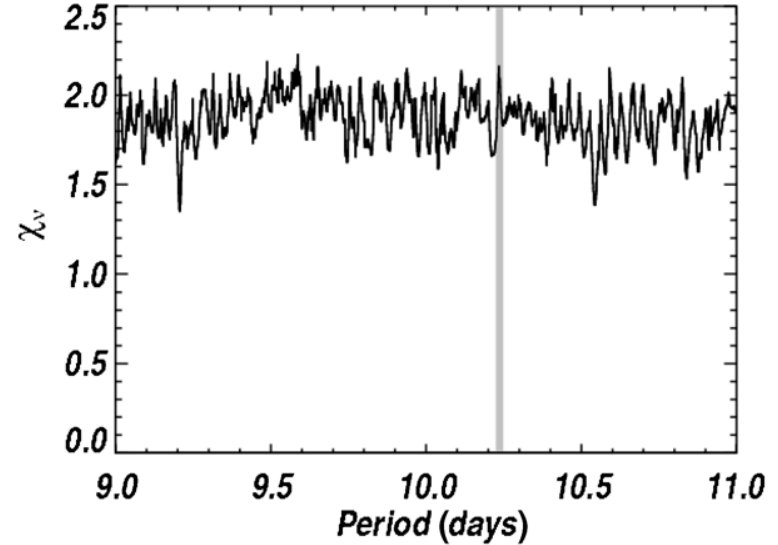

Figure 9. $\chi_{v}$ for a Keplerian fit to the real Keck velocities as a function of planet period. The eccentricity and semiamplitude have been frozen at the values reported by Endl et al. The value of $\chi_{v}$ does not approach unity near $P=10.2336$ days, as would be expected if the planet was present. The narrow shaded band shows $\pm 3 \sigma$ around the Endl et al. period.

Figure 9 shows $\chi_{v}$ for Keplerian fits to the real Keck-HIRES velocities for periods in the range of 9-11 days. The narrow shaded band highlights $\pm 3 \sigma$ around the Endl et al. period. Over the whole range of periods, $\chi_{v}$ is too large to be consistent with $\chi_{v} \approx 1$, as found for the synthetic Keck velocities. We conclude that the real Keck velocities are incompatible with the $K=11.7 \mathrm{~m} \mathrm{~s}^{-1}$ planet described by Endl et al. for periods in this range.

To estimate the largest semiamplitude $K$ allowed by the Keck velocities for a $\sim 10$ day orbit, we again fit the Keck data with a Keplerian as in Figure 9, but this time allowed $K$ to float. Figure 10 shows the best-fit $K$ for a potential planet having orbital periods ranging from 9 to 11 days. At precisely 10.2366 days, $K=1.4 \mathrm{~m} \mathrm{~s}^{-1}$. Note that $K$ is a rapidly varying function of $P$ and fluctuates as high as $4 \mathrm{~m} \mathrm{~s}^{-1}$ within $\pm 3 \sigma$ of the best-fit period. The Keck velocities therefore rule out the Endl et al. hypothesis - a planet in a 10.2366 day orbit - to a level of about $K=4 \mathrm{~m} \mathrm{~s}^{-1}$. This is nearly a factor of three smaller than the Endl et al. claim ( $5 \sigma$ below their reported value for $K$ ).

\subsection{Other Periodicities}

The origin of the $7.4 \mathrm{~m} \mathrm{~s}^{-1}$ of rms scatter in the Keck velocities remains an open question and motivation for additional observations of GJ 176. One possibility-which is not strongly supported by the current data-is that the variability is caused by a planet with a period of 8.77 days, as suggested by the periodogram peak at that period (Figure 6, middle panel). Fitting the Keck velocities for a Keplerian orbit plus a linear trend yields a $\chi_{v}=0.88$ solution with $K=5.8 \mathrm{~m} \mathrm{~s}^{-1}, e=0.09$, $M \sin i=12 M_{\text {Earth }}$, and a linear trend of $1.2 \mathrm{~m} \mathrm{~s}^{-1} \mathrm{yr}^{-1}$. While tantalizing, we caution against the premature and unwarranted identification of this feature as a planet for several reasons. ${ }^{10}$

First, the 8.77 day signal is a weak component of the Keck velocities. The periodogram peak at that period (Figure 6, middle panel) has an amplitude of only $\sim 9$, comparable to the amplitude of the 10.24 day peak in the HET data. The value of $\chi_{v}$ for the Keplerian fit plus linear trend would seem to imply

\footnotetext{
10 After submitting this manuscript on 2008 May 26, Forveille et al. (2009) submitted a manuscript to A\&A describing the nondetection of the 10.24 day planet orbiting GJ 176 based on data from the HARPS spectrograph. Instead, they found an $M \sin i=8.4 M_{\text {Earth }}$ planet in an 8.78 day circular orbit, a confirmation of the candidate described in this section.
}

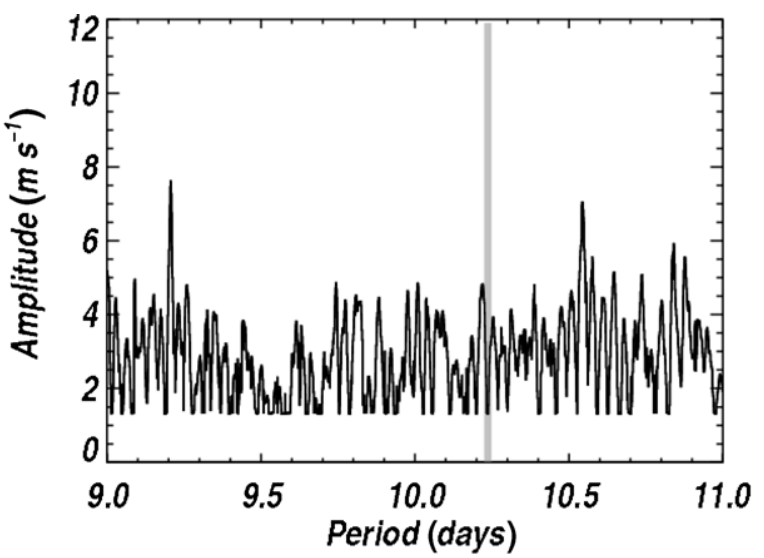

Figure 10. Best-fit semiamplitude, $K$, for a Keplerian fit to the real Keck velocities as a function of planet period. The eccentricity has been frozen at the value reported by Endl et al. The Keck data rule out a planet with a velocity semiamplitude greater than $4 \mathrm{~m} \mathrm{~s}^{-1}$ for periods within $3 \sigma$ of the Endl et al. value (the narrow shaded band shows this range).

a good fit, but is artificially low since the velocity uncertainties include $5 \mathrm{~m} \mathrm{~s}^{-1}$ of jitter added in quadrature (some of that jitter would be explained by the planet). Furthermore, we found a false alarm probability (FAP) of $12 \%$ for this fit, much too poor for a claimed detection by our standards. ${ }^{11}$ This FAP was calculated by performing a full search for a Keplerian plus a linear trend on 1000 mock Keck data sets that were scrambled with replacement.

The reality of a 8.77 day planet also depends on the statistical significance of the linear trend added to the Keplerian fit, without which the fit is poor. To evaluate this we calculated the FAP of the trend following the method of Wright et al. (2007). We compared $\chi_{v}$ for models with and without a trend, yielding $\Delta \chi_{v}=\chi_{v \text {,notrend }}-\chi_{v \text {,trend }}$. For the $P=8.77$ day Keplerian fit to the Keck data (with $5 \mathrm{~m} \mathrm{~s}^{-1}$ of jitter added to the errors), we find $\Delta \chi_{v}=0.25$. To test whether this reduction in $\chi_{v}$ could have arisen by chance fluctuations alone, we computed $\Delta \chi_{v}$ for a large ensemble of data sets, each with the scrambled residuals to the 8.77 day Keplerian fit without a trend. Using this method, we find FAP $=10 \%$ for the linear trend to the 8.77 day fit.

It is also worth noting that the $P=8.77$ day signal is not detected in the HET data. The periodogram amplitude near this period is less than 3 , well below the noise floor.

\section{DISCUSSION}

Precision Doppler velocity measurements by Keck-HIRES did not detect the claimed planet around GJ 176 despite a clear ability to do so. The presence of this planet thus seems unlikely and is called into question.

The Keck measurements impose a limit of $K \leqslant 4 \mathrm{~m} \mathrm{~s}^{-1}$ (a factor of 3 smaller than the reported value) for periods within 0.01 day $( \pm 3 \sigma)$ of 10.2366 days. Various tests quantitatively suggest that the Keck measurements would have easily revealed the planet, if present. We remain puzzled by the discrepancy between the reported HET-HRS results and the velocities obtained with Keck-HIRES, and welcome suggestions to resolve the inconsistency.

Endl et al. calculated a false alarm probability of $0.04 \%$ for the GJ 176 companion based on the HET data (via bootstrap reshuffling). In light of the Keck data, their calculation appears

\footnotetext{
11 Typically, we require FAP $\leqslant 1 \%$ or $0.1 \%$ to claim a detection (Marcy et al. 2005; Wright et al. 2007)
} 
to have significantly underestimated the true FAP. While their method of calculating the FAP-the fraction of scrambled data sets whose maximum amplitude periodogram peak is larger than said peak in the unscrambled set-is adequate to identify and flag candidate planets for further analysis, it can significantly underestimate the true FAP since it only detects the largest Fourier component of a possible Keplerian fit. Plausible false alarm orbital solutions with modest to high eccentricity can be easily missed, especially in cases with relatively few observations and large velocity uncertainties, leading to an underestimate of the FAP. A more conservative FAP calculation includes all false alarms with believable orbital characteristics, not just the ones with parameters similar to the orbit being tested (i.e., circular or nearly so). Based solely on the HET data and using a more conservative estimate of FAP based on the $\chi_{v}$ statistic for a Keplerian fit, we find FAP $=26 \%$ (256/ 1000 trials), an insecure detection. As in Section 2, this FAP calculation compares full Keplerian fits of the unscrambled HET data set and versions scrambled with replacement. While many of the false alarms are highly eccentric Keplerian solutions that might be dismissed as noise (50\% of the false alarms have $e>0.7$ ), a significant number of moderate eccentricity false alarm solutions remain (13\% of the false alarms have $e<0.5$ ). This FAP is insensitive to the inclusion of a linear trend with the Keplerian fit. We conclude that the HET velocities, evaluated independently, do not robustly support the Endl et al. claim of a 10.24 day planet around GJ 176 .

The sources of the velocity variability seen in GJ 176, and their relative strengths, remain unknown. To date, we do not see convincing evidence for temporal coherence (persisting for years) in the velocities. On the whole, the $9.8 \mathrm{~m} \mathrm{~s}^{-1} \mathrm{rms}$ scatter in the HET data is consistent with the range of scatter observed in the 90 stars in the HET M dwarf survey, most recently reported as rms $=8.3 \pm 3.9 \mathrm{~m} \mathrm{~s}^{-1}$ (Endl et al. 2006). While the Keck velocities have a slightly smaller rms scatter of $7.4 \mathrm{~m} \mathrm{~s}^{-1}$, this difference is not statistically significant for the limited number of observations in each set.

While we believe the 10.24 day planet has been ruled out by the Keck data, we will continue to observe GJ 176 to test the hypothesis of the 8.77 day planet (Section 2.3). A significant number of additional Keck observations, particularly on consecutive nights, are needed before such a determination can be made.

As Doppler velocity surveys begin to probe the observationally challenging realm of Neptune to Earth-mass planets, robust detections require independent confirmation and continued improvement in velocity precision as demonstrated by long-term stable stars. Such safeguards are essential for the credibility of the profound and extraordinary claims of the existence of other worlds.

The authors wish to extend special thanks to those of Hawaiian ancestry on whose sacred mountain of Mauna Kea we are privileged to be guests. Without their generous hospitality, the Keck observations presented herein would not have been possible. A.W.H. gratefully acknowledges support from a Townes Fellowship at the Space Sciences Laboratory at U. C. Berkeley. S.S.V. acknowledges NSF grant AST-0307493.

\section{REFERENCES}

Butler, R. P., Marcy, G. W., Williams, E., McCarthy, C., Dosanjh, P., \& Vogt, S. S. 1996, PASP, 108, 500

Butler, R. P., Vogt, S. S., Marcy, G. W., Fischer, D. A., Wright, J. T., Henry, G. W., Laughlin, G., \& Lissauer, J. J. 2004, ApJ, 617, 580

Endl, M., Cochran, W. D., Kürster, M., Paulson, D. B., Wittenmyer, R. A., MacQueen, P. J., \& Tull, R. G. 2006, ApJ, 649, 436

Endl, M., Cochran, W. D., Tull, R. G., \& MacQueen, P. J. 2003, AJ, 126, 3099

Endl, M., Cochran, W. D., Wittenmyer, R. A., \& Boss, A. P. 2008, ApJ, 673, 1165

Forveille, T., et al. 2009, A\&A, 493, 645

Goldreich, P., Lithwick, Y., \& Sari, R. 2004, ApJ, 614, 497

Ida, S., \& Lin, D. N. C. 2005, ApJ, 626, 1045

Ida, S., \& Lin, D. N. C. 2008, ApJ, 673, 487

Kennedy, G. M., Kenyon, S. J., \& Bromley, B. C. 2006, ApJ, 650, L139

Marcy, G. W., Butler, R. P., Vogt, S. S., Fischer, D. A., Henry, G. W., Laughlin, G., Wright, J. T., \& Johnson, J. A. 2005, ApJ, 619, 570

Tull, R. G. 1998, in Proc. SPIE 3355, Optical Astronomical Instrumentation, ed. S. D’Odorico (Bellingham, WA: SPIE), 387

Vogt, S. S., et al. 1994, in Proc. SPIE 2198, Instrumentation in Astronomy VIII, ed. D. L. Crawford \& E. R. Craine (Bellingham, WA: SPIE), 362

Wright, J. T., et al. 2007, ApJ, 657, 533 